\title{
Efecto de la combinación de clorhexidina y fluoruro de sodio sobre Streptococcus mutans en preescolares con manchas blancas.
}

\author{
Effect of the combination of chlorhexidine and sodium fluoride on Streptococcus mutans in preschools with \\ white spots. \\ Grascely Ayala ${ }^{1, a}$, María Álvarez ${ }^{1, b}$, Miguel Nuñez ${ }^{1, a}$
}

\section{RESUMEN}

Objetivos: Determinar el efecto de la combinación de los barnices diacetato de clorhexidina $1 \%$ y fluoruro de sodio 5 \% sobre Streptococcus mutans en saliva de niños de 3 a 5 años con manchas blancas. Material $\boldsymbol{y}$ métodos: Se incluyeron 45 niños, divididos en 3 grupos: combinación clorhexidina $1 \%$ y fluoruro de sodio $5 \%$ $(\mathrm{n}=15)$, fluoruro de sodio $5 \%(\mathrm{n}=15)$ y placebo $(\mathrm{n}=15)$. Después de 8 semanas se evaluó microbiológicamente. Resultados: Todos los grupos redujeron significativamente los $S$. mutans, combinación clorhexidina $1 \%$ y fluoruro de sodio $5 \%$ (15.267 \pm 9.816; p < 0.05); fluoruro de sodio $5 \%$ (16.267 \pm 7.146 ; p < 0.05); y placebo (9.467 \pm 9.326; p < 0.05). Conclusiones: La aplicación combinada diacetato de clorhexidina $1 \%$ y fluoruro de sodio $5 \%$ es efectiva para reducir los $S$. mutans en la saliva de los niños.

PALABRAS CLAVE: Clorhexidina, fluoruro de sodio, streptococcus mutans.

\section{SUMMARY}

Objectives: To determine the effect of the combination chlorhexidine diacetate $1 \%$ and sodium fluoride varnish $5 \%$ on Streptococcus mutans in the saliva of children 3 to 5 years with white stain. Methods: Were included 45 children, divided into 3 groups: combined chlorhexidine $1 \%$ and sodium fluoride $5 \%(\mathrm{n}=15)$, sodium fluoride $5 \%(\mathrm{n}=15)$ and placebo $(\mathrm{n}=15)$. After 8 weeks was evaluated microbiologically. Results: All groups significantly reduced the $S$. mutans, combination sodium fluoride $5 \%$ and chlorhexidine $1 \%$ (15.267 \pm 9.816 ; p $<0.05)$; sodium fluoride $5 \%(16.267 \pm 7.146 ; \mathrm{p}<0.05)$; and placebo $(9.467 \pm 9.326 ; \mathrm{p}<0.05)$. Conclusions: The combined application of chlorhexidine diacetate $1 \%$ and sodium fluoride $5 \%$ is effective reducing of $S$. mutans in saliva of children.

KEY WORDS: Chlorhexidine, varnishes, sodium fluoride, streptococcus mutans.

\footnotetext{
${ }^{1}$ Facultad de Odontología, Universidad Nacional Mayor de San Marcos. Lima, Perú.

${ }^{a}$ Cirujano Dentista.

${ }^{\text {b} C i r u j a n o ~ d e n t i s t a . ~ E s p e c i a l i s t a ~ e n ~ O d o n t o p e d i a t r i ́ a . ~ M a g i ́ s t e r ~ e n ~ O d o n t o e s t o m a t o l o g i ́ a ~ e n ~ S a l u d ~ P u ́ b l i c a . ~ D o c t o r a ~ e n ~ C i e n c i a s ~ d e ~ l a ~ S a l u d . ~}$
} 


\section{INTRODUCCION}

La caries dental sigue siendo un problema de salud prioritario en el país en niños de todas las edades. La clorhexidina y el flúor actúan de diferentes maneras en la prevención y control de la caries dental. El flúor tiene los tres mecanismos conocidos para ejercer su función cariostática: reducir la solubilidad del esmalte, remineralizar y su actividad antimicrobiana, mientras la clorhexidina solo actúa como antimicrobiano; su combinación y/o mixtura en altas concentraciones pueden producir un efecto bactericida frente a $S$. $m u$ tans, la principal bacteria responsable de la caries (1), los más beneficiados con esta combinación son los niños con alto riesgo de caries.

Jentsh et al., y Almaz et al., refieren que Twetman y Petersson realizaron múltiples estudios sobre ambos barnices, demostrando que la clorhexidina es eficaz aplicado con una frecuencia de intervalo corto $(1,2)$; pero este no tiene el mismo efecto sobre el $S$. $m u$ tans comparado a la mezcla de clorhexidina y flúor (3). Así también, al compararlo con el fluorsilano y el biofluoruro, la combinación de flúor y clorhexidina permitió que la biopelícula tuviera mayor efecto inhibidor frente el S. mutans (4). En nuestro país, tanto la clorhexidina como la mixtura de fluoruro-clorhexidina redujeron los niveles de $S$. mutans en la saliva de escolares con alto riesgo de caries (5). Al comparar la mixtura fluoruro clorhexidina frente al fluorsilano ambos obtuvieron valores de $S$. mutans muy por debajo de las puntuaciones considerados como nivel bajo $<10000$ o $10^{5} \mathrm{UFC} / \mathrm{ml}$ (unidades formadoras de colonia por mililitro) (6).

Actualmente no hay un control de fluorización ni mucho menos datos epidemiológicos que avalen su éxito. Si se indicaría estratégicamente la aplicación tópica de barnices fluorados y antimicrobianos de forma periódica en todos los niños, se lograría prevenir la enfermedad de la caries dental.

El objetivo del presente trabajo fue determinar el efecto de la combinación de los barnices diacetato de clorhexidina $1 \%$ y fluoruro de sodio $5 \%$ sobre Streptococcus mutans en saliva, en niños de 3 a 5 años con manchas blancas.

\section{MATERIAL Y MÉTODOS}

Se realizó un estudio piloto inicial con 15 niños de 3 a 5 años de edad que se atendieron en servicio de odontopediatría del Hospital Nacional Docente Madre Niño San Bartolomé, elegidos aleatoriamente y que cumplían los criterios de inclusión, al procesar los resultados de la evaluación se determinó que era necesario una muestra de 45 niños divididos en 3 grupos. Solo los niños cuyos padres firmaron el consentimiento informado, participaron. Inicialmente se preparó una charla preventivo promocional sobre la salud oral en niños, donde se educó y motivó con una técnica de cepillado a padres e hijos. Se evaluó el índice de higiene oral a los niños y este fue controlado hasta homogenizar la muestra.

La primera muestra salival del paciente se tomó en horario de la mañana, el cual se cultivó en un medio agar mitis salivarius con bacitracina, obteniendo unidades formadoras de colonias (UFC) de S. mutans que fueron contabilizadas para su registro. Al inicio de cada aplicación recibían un cepillado de dientes con pasta profiláctica, posteriormente se realizaba un aislamiento relativo donde se secaba la superficie del diente: combinación clorhexidina $1 \%$ y fluoruro de sodio $5 \%$ (2 veces fluoruro, 1 vez clorhexidina); fluoruro de sodio $5 \%$ (3 veces fluoruro); y placebo (3 veces agua destilada) en un período de 10 días.

Después de 8 semanas, se recogió la segunda muestra salival, por ello se citó a los pacientes a la misma hora que se tomó la muestra inicial; masticaron un cubo de parafina para estimular la saliva durante 2 minutos; escupieron toda la saliva en un frasco recolector estéril; y estas fueron conservadas en un cooler con hielo, tratando de mantener bajas temperaturas y ser transportadas hasta el laboratorio. Para el cultivo agar mitis salivarius con bacitracina se depositó una dilución de la muestra de saliva de los niños, se incubó en una jarra de anaerobiosis bajo $37^{\circ} \mathrm{C}$ durante 48 horas, y posteriormente se realizó el recuento de colonias típicas formadas por el $S$. mutans en las placas.

Los datos recolectados fueron registrados en una ficha, estos fueron codificados para un mejor procesamiento, se utilizó el software SPSS Statistics 20.0 para el análisis estadístico, tanto la prueba t para las muestras relacionadas y ANOVA para las muestras independientes. 


\section{RESULTADOS}

Todos los grupos redujeron significativamente los $S$. mutans después de hacer el tratamiento con los barnices, incluso en muchas de las placas petri no se formó ni una sola colonia (Figura 1). La combinación clorhexidina $1 \%$ y fluoruro de sodio $5 \%$ inicialmente $21.133 \pm 9.797$ y después $5.867 \pm 6.266$ (Tabla 1); el fluoruro de sodio $5 \%$ en un inicio $17.800 \pm 7.711$ y después $1.533 \pm 2.774$ (Tabla 2); el placebo antes
$14.000 \pm 11.607$ y después $4.533 \pm 5.755$ (Tabla 3).

Al evaluar según el efecto de reducción de $S$. mutans, el mayor efecto lo obtuvo el fluoruro de sodio $5 \%$ (16.267 $\pm 7.146 ; \mathrm{p}<0,05)$, luego la combinación clorhexidina $1 \%$ y fluoruro de sodio $5 \%$ (15.267 \pm 9.816; $\mathrm{p}<0.05)$; y el placebo (9.467 \pm 9.326 ; $\mathrm{p}<0.05$ ); pero al compararlos entre sí no existió significancia (p: 0.087) (Tabla 4 y Gráfico 1).
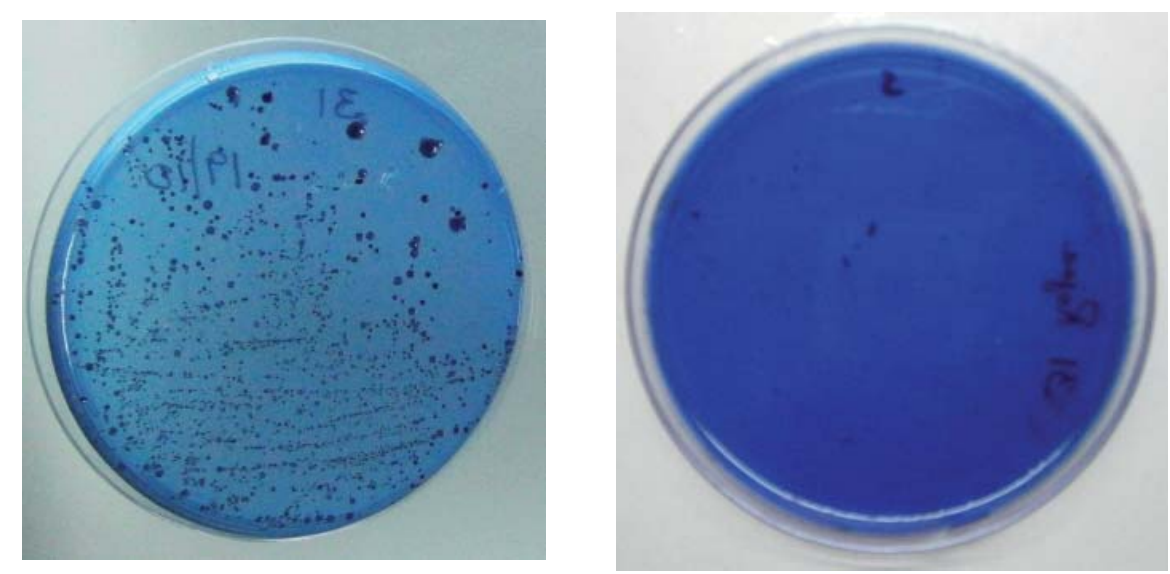

Figura 1. Cultivos microbiológicos de un niño que recibió la combinación de Fluoruro de sodio $5 \%$ y Clorhexidina $1 \%$ (antes y final)

Tabla 1. Efecto de la combinación clorhexidina $1 \%$ y fluoruro de sodio 5 \% sobre S. mutans

Diferencias relacionadas

Valor

$\mathrm{p}$

\begin{tabular}{lcccc} 
& Media & Desv. Tip. & IC 95 \% & \\
\hline $\begin{array}{l}\text { Antes de tratamiento } \\
\text { (S. mutans) }\end{array}$ & 21.133 & 9.797 & $15.708-26.559$ & 0.000 \\
$\begin{array}{l}\text { Final de tratamiento } \\
\text { (S. mutans) }\end{array}$ & 5.867 & 6.266 & $2.396-9.337$ & \\
\hline
\end{tabular}

* Prueba t para muestras relacionadas 
Tabla 2. Efecto de fluoruro de sodio 5\% sobre S. mutans

\begin{tabular}{|c|c|c|c|c|}
\hline & \multicolumn{3}{|c|}{ Diferencias relacionadas } & \multirow{2}{*}{$\begin{array}{c}\text { Valor } \\
\mathrm{p}\end{array}$} \\
\hline & Media & Desv. Tip. & IC $95 \%$ & \\
\hline $\begin{array}{l}\text { Antes de tratamiento } \\
(S . \text { mutans })\end{array}$ & 17.800 & 7.711 & $13.530-22.070$ & 0.000 \\
\hline $\begin{array}{l}\text { Final de tratamiento } \\
\text { (S. mutans) }\end{array}$ & 1.533 & 2.774 & $0.003-3.070$ & \\
\hline
\end{tabular}

* Prueba t para muestras relacionadas

Tabla 3. Efecto de placebo sobre S. mutans

Diferencias relacionadas

Valor

$\mathrm{p}$

Media Desv. Tip. $\quad$ IC $95 \%$

\begin{tabular}{|c|c|c|c|c|}
\hline $\begin{array}{l}\text { Antes de tratamiento } \\
\text { (S. mutans) }\end{array}$ & 14.000 & 11.607 & $7.572-20.428$ & 0.002 \\
\hline $\begin{array}{l}\text { Final de tratamiento } \\
\text { (S. mutans) }\end{array}$ & 4.533 & 5.755 & $1.346-7.721$ & \\
\hline
\end{tabular}

* Prueba t para muestras relacionadas

Tabla 4. Comparación de efectos de combinación clorhexidina $1 \%$ y fluoruro de sodio 5 \%, fluoruro de sodio $5 \%$, y placebo sobre S. mutans

\begin{tabular}{|c|c|c|c|c|}
\hline & \multicolumn{3}{|c|}{ Diferencias relacionadas } & \multirow{2}{*}{ Sig } \\
\hline & Media & Desv. Tip. & IC $95 \%$ & \\
\hline $\begin{array}{l}\text { Efecto de combinación Clorhexidina } \\
1 \% \text { y fluoruro de sodio } 5 \%\end{array}$ & 15.267 & 9,816 & $9.831-20.703$ & 0.087 \\
\hline Efecto de fluoruro de sodio 5\% & 16.267 & 7,146 & $12.309-20.224$ & \\
\hline Efecto de Placebo & 9.467 & 9.326 & $4.302-14.631$ & \\
\hline
\end{tabular}

* Prueba ANOVA para muestras independientes 


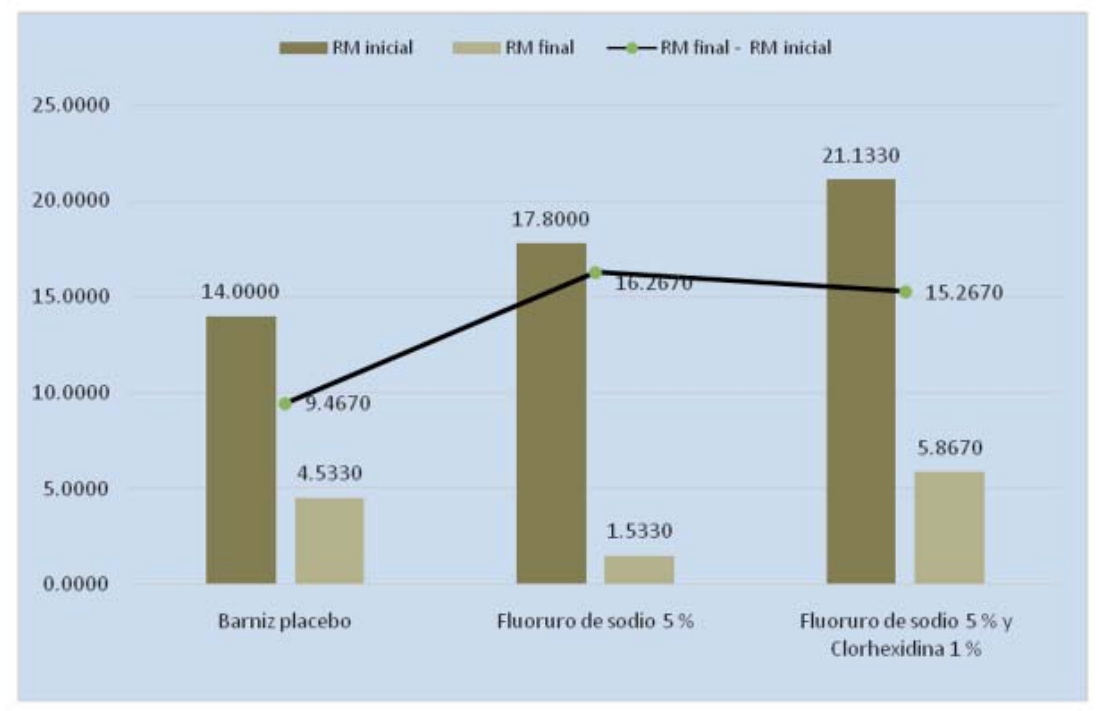

Gráfico 1. Comparación de efectos de combinación clorhexidina $1 \% \mathrm{y}$ fluoruro de sodio $5 \%$, fluoruro de Sodio $5 \%$, y placebo sobre S. mutans

\section{DISCUSIÓN}

En salud pública el método ideal para combatir la caries dental es el cepillado de dientes con una pasta dental con flúor porque es práctico, de bajo costo, de gran alcance y está aceptado culturalmente. En varias revisiones sistemáticas recomiendan indicar a todos los paciente el uso de la pasta dental fluorada (7-11). Frencken et al., recomiendan que el nivel de flúor en las pastas dentales para los niños pequeños (menos de 6 años de edad) deba ser por debajo de las 1000 ppm (10).

En la eficacia anticaries de las pastas dentales fluoradas, existen tres factores importantes a considerar: concentración, frecuencia de cepillado y enjuague post-cepillado (11); existe un orden de factores que influencian el efecto preventivo de la caries usando el cepillado dental, el principal factor es el empleo de un dentífrico fluorado que representa un $24 \%$ de efectividad; un segundo factor fundamental es que el cepillado se realice 2 veces al día y esto influye un 14 $\%$; otro factor influyente en un 11 \% es la supervisión del cepillado; además el cepillado más topificaciones adicionales de flúor genera un $10 \%$ de efectividad, y finalmente que la pasta dental contenga entre 1450 - 1500 ppm reduce eficazmente un $8 \%$ la caries (1215). En una revisión sistemática se propuso examinar el efecto de la fluorización tópica adicional compa- rada con el uso de la pasta dental fluorada y se halló una modesta reducción de caries en comparación, solamente al dentífrico (16). En nuestra investigación los participantes recibieron una charla preventivo promocional de higiene oral con la recomendación del uso de una pasta dental fluorada para niños; y los que recibieron placebo redujeron los $S$. mutans, dado que los niños estaban expuestos al flúor diariamente.

La misma efectividad también se encontraron en los estudios de Pinar et al., y Álvarez et al., quienes tuvieron grupos controles usando solo pastas dentales fluoradas para compararlos con otros agentes quimioterapéuticos, logrando reducir los niveles de microorganismos y caries dental $(4.6,21)$.

El flúor es el principal agente de cualquier programa completo de prevención para el control de caries. Se resalta al flúor barniz como un tópico efectivo en la prevención y control de la caries, en la revisión de cinco estudios disponibles se demostró una fracción preventiva para dentición temporal de 33 \% estableciéndose que se aplicará en superficies de riesgo a desarrollar lesiones de caries en intervalos de 3 a 6 meses (17). En otro estudio, se evaluó el efecto de la aplicación semianual de barniz fluorado en el desarrollo de la caries en dientes deciduos en niños de 4 años de edad y encontraron un $30 \%$ de reducción de caries. Otra característica del flúor barniz es que 
es más efectivo cuando se aplica en superficies que presentan lesiones incipientes que en esmalte sano (18). Cuando se estudió el efecto del barniz fluorado en lesiones cariosas incipiente, presente en dientes deciduos recién erupcionados, se demostró que dos aplicaciones de barniz fluorado es efectivo, deteniendo la lesión cariosa activa del esmalte, sobre todo en niños con alto riesgo de caries (19). En nuestro estudio el fluoruro de sodio $5 \%$ tuvo un mayor comportamiento antimicrobiano. Anderson et al., refieren que Weintraub et al., mostraron la misma eficacia cuando aplicó este mismo flúor con diferentes frecuencias de tratamiento, pero que asociados a un previo asesoramiento pediátrico redujeron la incidencia de caries en la primera infancia (20). Y cuando se comparó entre sí a los productos de flúor existentes en el mercado, se concluyó que el Duraphat ${ }^{\circledR}$ tiene mayor eficacia en la reducción de un 58 \% en profundidad de la lesión de caries de esmalte en dientes deciduos con una diferencia significativa en comparación con el grupo control (sin pasta dental fluorada), pasta de dientes para niños 500 ppm y marcas variadas de flúor (21). Sin embargo otros estudios no encontraron significancia estadística del flúor como agente antimicrobiano $(5,6,22)$.

En revisiones bibliográficas se ha indicado que la clorhexidina barniz puede considerarse una opción a corto plazo para controlar la caries en personas con alto riesgo de caries que tiene a su vez altos recuentos de bacterias (10). Al compararse la efectividad de las presentaciones de la clorhexidina en el tiempo, concluyeron que la presentación de clorhexidina barniz después de tres meses mantuvo los niveles de S. mutans reducidos (2). También se ha establecido que la clorhexidina barniz tiene mayor eficacia tras ser aplicado en un tratamiento intensivo de 3 dosis en un período de 10 días, lográndose una significativa reducción de los $S$. mutans ( $\mathrm{p}<0.01$ ) en un plazo mayor (1). De igual modo se afirmó que las aplicaciones constantes de la clorhexidina barniz son más eficaces que una sola aplicación de este, para la disminución en la incidencia de caries $(23,24)$. Finalmente hay evidencia que en ausencia de una adecuada limpieza dental, el barniz de clorhexidina proporciona efectos beneficiosos en pacientes que lo necesitan (10).

La asociación del flúor y clorhexidina han sido evaluadas constantemente, en un estudio que duro 3 años se encontró efectividad de la combinación de fluorsilano $1 \%$ y clorhexidina $1 \%$ en la reducción de caries interproximal (22). Esta eficacia como agente anticariogénico, también lo tiene frente a microorganismos como el S. mutans, por lo que su asociación también tiene poder antimicrobiano. Otros autores encontraron eficacia en la reducción de $S$. mutans al aplicar la mezcla de clorhexidina $1 \%$ y fluorsilano $1 \%$ al compararlo con un grupo que inicialmente recibió clorhexidina 1 \% (3). También Díaz y Álvarez encontraron valores reducidos en el recuento microbiano de S. mutans al finalizar los tratamientos de aplicación, a diferencia de los otros agentes barnices, quienes si disminuían los niveles, pero no mostraban significancia estadística $(5,6)$. Todos estos valores fueron fundamentados por Donelly y Larjava quienes mediante su revisión bibliográfica, según Pacheco, definió al flúor y clorhexidina como agentes totalmente diferentes, que al combinarlos no pierden sus propiedades; y su asociación debe ser de uso en pacientes con potenciales riesgos de caries, por ello recomienda a los dentistas utilizar la combinación reconociendo su importancia y secuencia de tratamiento (25). Nuestro grupo de niños que recibió esta combinación tuvo un resultado efectivo similar, concluyéndose que la aplicación combinada de diacetato de clorhexidina 1 $\%$ y fluoruro de sodio $5 \%$ es efectiva para reducir $S$. mutans en la saliva de los niños.

\section{Correspondencia:}

Grascely Ayala-Gonzales

Correo electrónico: grascely.ayala@gmail.com

\section{REFERENCIAS BIBLIOGRAFICAS}

1. Jentsh H, Eckert F, Eschrich K, Stratul S, Kneist D. Antibacterial action of chlorhexidine/thymol containing varnishes in vitro and in vivo. Int J Dent Hyg. 2014;12(3):168-73.

2. Almaz M, Sonmez I, Okte Z, ObaA. Efficacy of a sugar-free herbal lollipop for reducing salivary Streptococcus mutans levels: randomized controlled trial. Clin Oral Investig. 2016;214:161-7.

3. Sajjan PG, Nagesh L, Sajjanar M, Reddy S, Venkatesh U. Comparative evaluation of chlorhexidine varnish and fluoride varnish on plaque streptococcus mutans count-an in vivo study. Int J Dent Hyg. 2013;11(3):191-7.

4. Pinar Erdem A, Sepet E, Kulekci G, Trosola S, Guven Y. Effects of two fluoride varnishes an one fluoride/ chlorhexidine varnish on Streptococcus mutans and Streptococcus sobrinus biofilm formation in vitro. Int 
J Med Sci. 2012;9(2):129-36.

5. Díaz A. Estudio comparativo del efecto de las aplicaciones de barniz flúor y/o clorhexidina sobre algunos factores clínicos microbiológicos de riesgo a caries dental. Tesis de Maestría. Lima: Universidad Nacional Mayor de San Marcos; 2005.

6. Álvarez M. Uso de agentes quimioterapéuticos para el control y regresión de manchas blancas de pacientes preadolescentes. Tesis de Maestría. Lima: Universidad Nacional Mayor de San Marcos; 2007.

7. Naidy R, Nunn J, Irwin JD. The effect of motivational interviewing on oral healthcare knowledge, attitudes and behavior of parents and caregivers of preschool children: an exploratory clusted randomized controlled study. BMC oral health. 2015;15(1):101-16.

8. Espinoza E, Pachas M. Programas preventivos promocionales de salud bucal en el Perú. Rev Estomatol Herediana. 2014;23(2):101-8.

9. Ministerio de Salud. Módulo de promoción de la salud bucal. Lima: Ministerio de Salud; 2013.

10. Frencken J, Peters M, Manton D, Leal S, Gordan V, Eden E. Minimal intervation Dentistry (MID) for managing dental caries - a review. Int Dent J. 2012;62(5):223-43.

11. Ministerio de Salud. Guía clínica AUGE Salud Oral integral para niños y niñas de 6 años. Santiago: Ministerio de Salud; 2013.

12. Vermaire J, Poorterman J, Van Herwijnen L, Van Loveren C. A three-year randomized controlled trial in 6-year-old children on caries-preventive strategies in a general dental practice in the Netherlands. Caries Res. 2014;48(6):524-33.

13. Wolff M, Hill R, Wilson M, Hirsch S, Dasanayake A. Nationwide 2.5 year school-based public health intervention program designed to reduce the incidence of caries in children of Grenada. Caries Res. 2016;50(1):68-77.

14. Dos Santos APP, Nadanovsky P, De Oliveira B. A systematic review and meta-analyses of the effects of fluoride toothpastes on the prevention of dental caries in the primary dentition of preschool children. Community Dent Oral Epidemiol. 2013;41(1):1-12.

15. Zhou S, Zhou J, Watanabe S, Watanabe K, Wen L, Xuank. In vitro study of the effects of fluoride-releasing dental materials on remineralization in an enamel erosion model. J Dent. 2012;40(3):255-63.

16. Ten Cate JM. Contemporany perspective on the use of fluoride products in caries prevention in children and adolescents.Br Dent J. 2013;214:161-67.

17. Ministerio de Salud. Protocolo de cepillado y aplicación comunitaria de barniz de flúor para intervención en párvulos. Santiago: Ministerio de Salud; 2012.

18. Cho S, Kim J, Kim J. Remineralization effects on the desmineralized enamel of primary teeth by fluoride varnish. J Korean Acad Pediatr Dent. 2016;43(1):44-
50.

19. Chou R, Cantor A, Zakher B, Michell JP, Pappas M. Preventing dental caries in children $<5$ years: systematic review updating USPSTF recommendation. Pediatrics. 2013;132(2):332-50.

20. Anderson M, Dahllöf G, Tewtman S, Jansson L, Bergenlid A, Gridefford M. Effectiveness of early preventive intervention with semiannual fluoride varnish application in toddlers living in high-risk areas: a stratified cluster randomized controlled trial.Caries Res. 2016;50(1):17-23.

21. De Carvalho F, Vieira B, Santos R, Lopes P, De Lima B. In vitro effects of nano-hydroxyapatite paste on initial enamel carious lesions. Pediatr Dent. 2014;36(3):85-9.

22. Flamee S, Gizani S, Caroni C, Papagiannoulis L, Twetman S. Effect of a chlorhexidine/thymol and a fluoride varnish on caries development in erupting permanent molars: a comparative study. European Archives of Pediatric Dentistry. 2015;16(6):449-54.

23. Gupta A, Marya C, Dahiya V, Bhatia H, Dhingra S. Preventing dental caries in children: Indian scenario. Kathmandu Univ Med J. 2012;10(37):77-82.

24. Sumeera K, Mathew S, Hegde S, Muthy B, George J. Antimicrobial effects of the combination of chlorhexidine and fluoride varnish in young adult population. World Journal of dentistry. 2012;3(4):290-3.

25. Pacheco G. Reducción de la carga bacteriana oral en niños de séptimo de básica de la escuela Manuelita Saenz, utilizando flúor barniz, flúor fosfato acidulado, barniz de clorhexidina y clorhexidina en gel. Tesis de bachiller. Quito: Universidad Central del Ecuador; 2015.

Recibido : 18/03/2016

Aceptado: 12/05/2016 